\title{
Biologikatherapie von Rheumapatienten nach Krebsdiagnose
}

Pappas DA et al. Therapy with Biologic Agents After Diagnosis of Solid Malignancies: Results from the Corrona Registry. J Rheumatol 2019; 46: 1438-1444

Patienten mit rheumatoider Arthritis (RA) und zuvor behandelten soliden Tumoren können leitliniengemäß genauso behandelt werden, wie diejenigen ohne solche Vorgeschichte. Die Evidenz dieser Empfehlung ist aber begrenzt, weswegen Rheumatologen nach einer Krebsdiagnose zögern eine biologische Therapie zu beginnen oder fortzusetzen. In einer Registerstudie wurde daher die Anwendung von Biologika bei Patienten mit RA und Krebsdiagnose untersucht.

In die Studie wurden RA-Patienten des CORRONA-Registers zwischen März 2002 und März 2016 eingeschlossen, bei denen innerhalb von 12 Monaten im Follow-up ein solider Tumor bei mindestens einem Kontrollbesuch erstmals diagnostiziert wurde. Geschätzt 
wurde der Anteil von Patienten, die nach der Diagnose die Behandlung mit biologischen bzw. gezielt wirkenden, synthetischen, krankheitsmodifizierenden, antirheumatischen Medikamente (bDMARD bzw. tsDMARD) entweder fortsetzten oder begannen. Berechnet wurde die mediane Zeit bis zur Einleitung von bDMARD/tsDMARD nach der Diagnose und der Anteil, derjenigen die die biologische Behandlung in einem Zeitintervall von 6 Monaten einleiteten. Das CORRONA(Consortium of Rheumatology Researchers of North America) Register ist die, nach eigenen Angaben, weltweit größte prospektive RA-Kohortenstudie, an der sich 169 rheumatologische Zentren in 40 Staaten der USA beteiligen.

Von 42619 Patientinnen und Patienten mit RA, die zum Studienzeitpunkt in CORRONA eingeschlossen waren, hatten 934 Patienten nach der Registrierung einen soliden Tumor und von diesen erfüllten 880 Patienten das Kriterium von mindestens einem Kontrollbesuch innerhalb von 12 Monaten.
Die Gesamtnachbeobachtungszeit der auswertbaren 880 Patientinnen und Patienten erreichte 2585,6 Personenjahren. Der überwiegende Teil der Studienteilnehmer waren Frauen (67\%) im Durchschnittsalter von 66,6 Jahren, mit einer mittleren RA-Krankheitsdauer von 14,1 Jahren vor der Karzinomdiagnose. Die häufigsten Krebsarten waren Brustkrebs (21\%) und ein Melanom der Haut (9\%).

Von den Studienteilnehmern wurden 367 $(41,7 \%)$ innerhalb der 12 Monate vor der Krebsdiagnose mit bDMARDs bzw. tsDMARDs behandelt. Nach der Krebsdiagnose nahmen von diesen Patientinnen und $\mathrm{Pa}$ tienten 270 (30,7\%) diese Medikamente bei der ersten Visite nach der Diagnose ein, 44 (5\%) wechselten innerhalb von 36 Monaten die Wirkstoffe der Biologika und weitere 90 Patienten (10,2\%) begannen eine Therapie mit Biologika. Die Mehrzahl der Patientinnen und Patienten, die Biologika im Verlauf der Nachuntersuchung einnahmen, ver- wendeten eine Tumornekrosefaktor-Inhibitor (53,5\%).

\section{FAZIT}

In der Praxis wurde fast ein Drittel der untersuchten RA-Patienten unmittelbar nach der Krebsdiagnose mit systemisch wirkenden Medikamenten behandelt und ein beträchtlicher Prozentsatz der Patientinnen und Patienten startete innerhalb von 3 Jahren eine Therapie mit Biologika. Die Mehrzahl der bDMARD/ tsDMARD-Erstbehandlungen nach Krebsdiagnose erfolgte mit einem TNF-Hemmer.

Richard Kessing, Zeiskam 